15

ournal of

Mechanics of

Materials and Structures

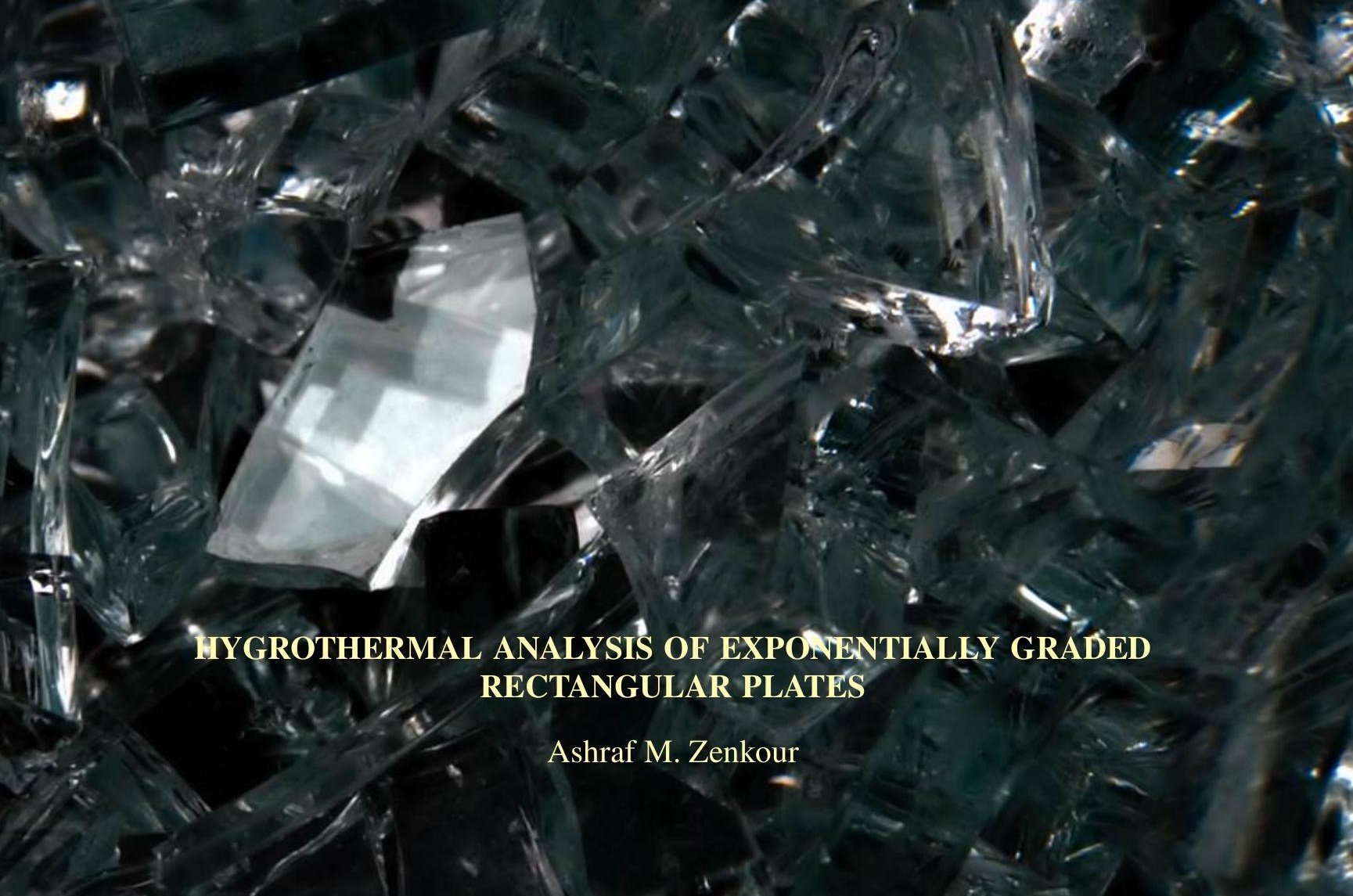

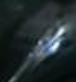

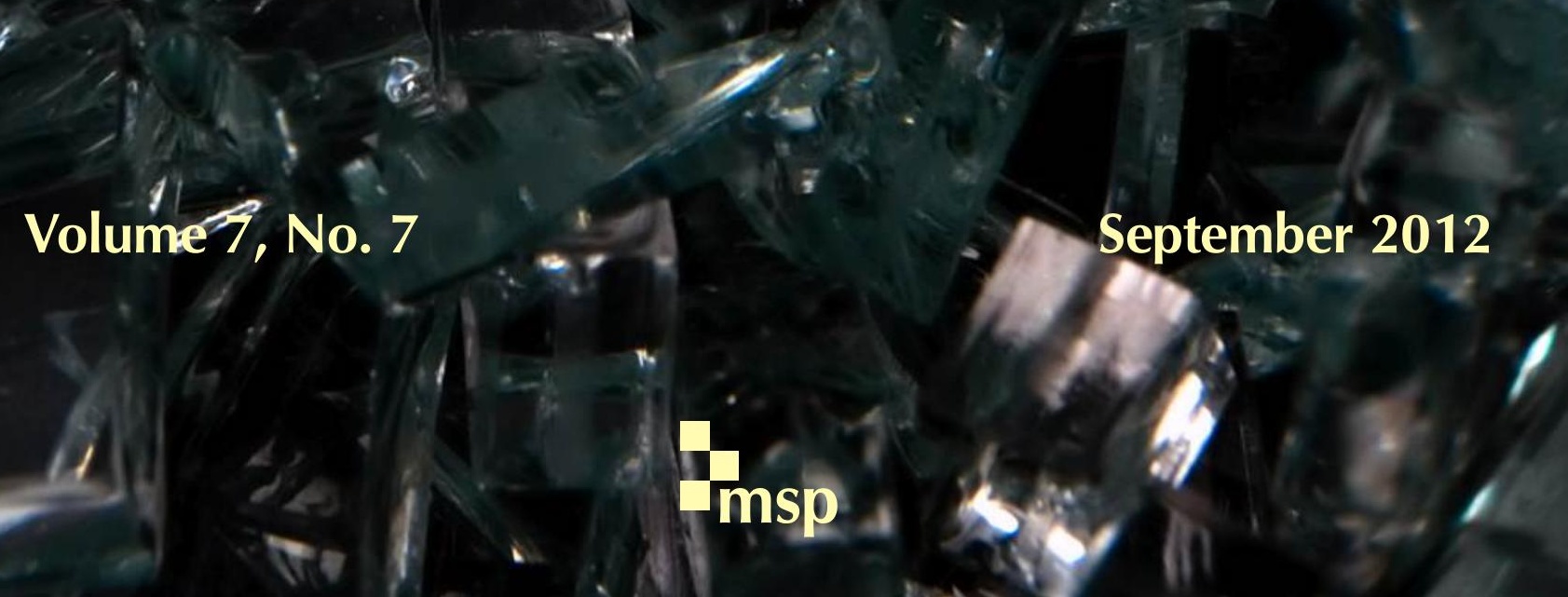




\title{
HYGROTHERMAL ANALYSIS OF EXPONENTIALLY GRADED RECTANGULAR PLATES
}

\author{
ASHRAF M. ZENKOUR
}

\begin{abstract}
The static characteristics of an exponentially inhomogeneous plate under a transverse uniform loading and exposed to hygrothermal conditions are studied. The elastic coefficients, thermal coefficient and moisture expansion coefficient of the plate are assumed to be exponentially graded in the thickness direction. The trigonometric shear deformation theory solution is presented. In this solution, the initial terms of a power series through the plate thickness are used for the displacements in addition to other trigonometric terms. The effect due to transverse shear deformations is included. A number of examples will be solved to illustrate the numerical results concerning bending response of homogeneous and exponentially graded rectangular plates subjected to hygrothermomechanical effects. The influences of temperature, moisture concentration, transverse shear deformation, plate aspect ratio, and the exponentially graded parameter on the bending response are investigated.
\end{abstract}

\section{Introduction}

The analysis of the rectangular plates subjected to moisture and temperature effects has been the subject of research interest of many investigators. Moisture and temperature may be distributed through the volume of the structure and may induce residual stresses and extensional strains. These residual stresses and extensional strains may also affect the gross performance of the structure. In particular, the bending characteristics, buckling loads and vibration frequencies can be modified by the presence of moisture, temperature or both. Therefore, to utilize the full potential of advanced structures, it will be necessary to analyze the effects of moisture and temperature in composite structural components.

The vibration characteristics of thick isotropic rectangular plates under an arbitrary state of initial stress were investigated in [Herrmann and Armenakas 1962; Brunelle and Robertson 1974; 1976]. Adams and Miller [1977], Ishikawa et al. [1978] and Strife and Prewo [1979] have studied the effect of environment on the material properties of composite materials and observed that it has significant effect on strength and stiffness of the composites. Therefore, there is a need to understand the behavior of composite structures subjected to hygrothermal conditions. Whitney and Ashton [1971] have used the classical laminate plate theory to study the hygrothermal effects on bending, buckling and vibration of composite laminated plates using the Ritz method and neglecting the transverse shear deformation. Pipes et al. [1976] have presented the distribution of in-plane stresses through the thickness of symmetric laminates subjected to moisture absorption and desorption. Yang and Shieh [1987] have considered the free vibration of antisymmetric cross-ply laminates in presence of a non-uniform initial stress, where the effects of transverse shear and rotary inertia were also included. Sai Ram and Sinha [1991; 1992] have studied the hygrothermal effects on the bending and free vibration behavior of laminated composite plates using

Keywords: hygrothermal conditions, rectangular plate, exponentially graded. 
the first-order shear deformation theory and employing finite element method. The effects of moisture and temperature on the deflections and stress resultants are presented for simply supported and clamped antisymmetric cross-ply and angle-ply laminates using reduced lamina properties at elevated moisture concentration and temperature. Lee et al. [1992] have studied the influence of hygrothermal effects on the cylindrical bending of symmetric angle-ply laminated plates subjected to uniform transverse load for different boundary conditions via classical laminated plate theory and von Karman's large deflection theory. The material properties of the composite are assumed to be independent of temperature and moisture variation. It has been observed that the classical laminated plate theory may not be adequate for the analysis of composite laminates even in the small deflection range.

Many studies, based on classical plate theory, of thin rectangular plates subjected to mechanical or thermal loading or their combinations as well as the hygrothermal effects are available in the literature [Whitney and Ashton 1971; Sai Ram and Sinha 1992]. However, studies of temperature and moisture effects on the bending of rectangular plates based on the shear deformation theories are limited in number, and all these studies assumed perfectly initial configurations [Pipes et al. 1976; Yang and Shieh 1987; Sai Ram and Sinha 1991; 1992]. The classical laminated plate theory and the first-order shear deformation plate theory are typical deformation theories for the analysis of laminated composite plates. The classical theory neglects the shear stresses while the first order theory assumes a constant transverse shear strain across the thickness direction, and a shear correction factor is generally applied to adjust the transverse shear stiffness for the static and stability analyses. However, some investigations showed that the bending and postbuckling responses of rectangular plates are sensitive to the choice of the shear correction factor.

To avoid the use of shear correction factor, various higher-order theories have been proposed to predict the bending response of rectangular plates. Shen [2001] has investigated the influence of hygrothermal environment on postbuckling behaviors of laminated plates based on Reddy's [1997] higher-order plate theory, considering the effects of temperature and moisture on the material properties. Patel et al. [2002] have studied the static and dynamic response of the thick laminated composite plates under hygrothermal environment based on a higher order theory. Rao and Sinha [2004] have studied the effects of moisture and temperature on the bending characteristics of thick multidirectional fibrous composite plates. The finite element analysis accounts for the hygrothermal strains and reduced elastic properties of multidirectional composites at an elevated moisture concentration and temperature. Deflections and stresses have been evaluated for thick multidirectional composite plates under uniform and linearly varying throughthe-thickness moisture concentration and temperature. Results reveal the effects of fiber directionality on deflection and stresses. Wang et al. [2005] have studied the response of dynamic interlaminar stresses in laminated composite plates with piezoelectric layers using an analytical approach. Benkhedda et al. [2008] have proposed an analytical approach to calculate the hygrothermal stresses in laminated composite plates, and took into account the change of mechanical characteristics due to moisture and temperature. In their study, the distribution of the transient in-plane stresses through the thickness of laminates is presented, whereas the transverse stresses were not taken into account. Lo et al. [2010] have developed a global-local higher order theory to study the response of laminated plates exposed to hygrothermal environment. Recently, Zenkour [2010] has presented a hygrothermal bending analysis for a functionally graded material (FGM) plate resting on elastic foundation.

From the literature reviewed, it can be found that research on local hygrothermal stresses of exponentially graded material (EGM) plates subjected to temperature and moisture effects seems to be lacking, 
which is the problem to be addressed in this paper. It is to be noted that, moisture and temperature have an adverse effect on the performance of composites. Stiffness and strength are reduced with the increase in moisture concentration and temperature. However, the discussion on the change of global and local response with respect to material variation due to elevated temperatures and moisture concentrations has been less reported in the published literature. In this article, a trigonometric shear deformation plate theory is developed to study the response of exponentially graded rectangular plates exposed to hygrothermal conditions. The present theory satisfies the continuity conditions of transverse shear stresses through the plate thickness. The analysis takes into account the change of material properties and a change in moisture concentrations through the plate thickness. Relationships between displacements/stresses and temperature or moisture concentrations have been studied under different hygrothermal conditions

The objective of this investigation is to present a general hygrothermal formulation for EGM plates using the sinusoidal shear deformation theory [Zenkour 2004a; 2004b; 2006; 2009]. The model has been developed within the phenomenological approach, where the effect of temperature and moisture has been taken into account at constitutive level. The governing partial differential equations are reduced to a set of coupled ordinary differential equations in the thickness direction. Numerical results for displacements and stresses are presented for homogeneous and EGM plates subjected to hygrothermomechanical effects. To make the study reasonably, displacements and stresses are given for different environment parameters and homogenization schemes as well as exponents in the power-law that describes through-the-thickness variation of the plate.

\section{Mathematical model}

Consider a rectangular plate of length $a$, width $b$ and thickness $h$ made of an exponentially graded material (EGM). The plate is subjected to a distributed transverse static mechanical load $q(x, y)$ and a temperature field $T(x, y, z)$ as well as a moisture concentration $C(x, y, z)$. The sinusoidal plate theory is presented for the small displacement and the corresponding small strains. The material properties $P$ of the EGM plate, such as Young's modulus and the thermal and moisture expansion coefficients are assumed to be functions of the thickness of the plate. The present plate is supported at four edges defined in the $(x, y, z)$ coordinate system with $x$ - and $y$-axes located in the middle plane $(z=0)$ and its origin placed at the corner of the plate as shown in Figure 1. An exponential relationship between the material property $\bar{P}$ and $z$ for the EG plate is assumed as in [Zenkour 2005]:

$$
\bar{P}=\bar{P}(z)=\bar{P}_{0} e^{-\eta(z / h)^{k}},
$$
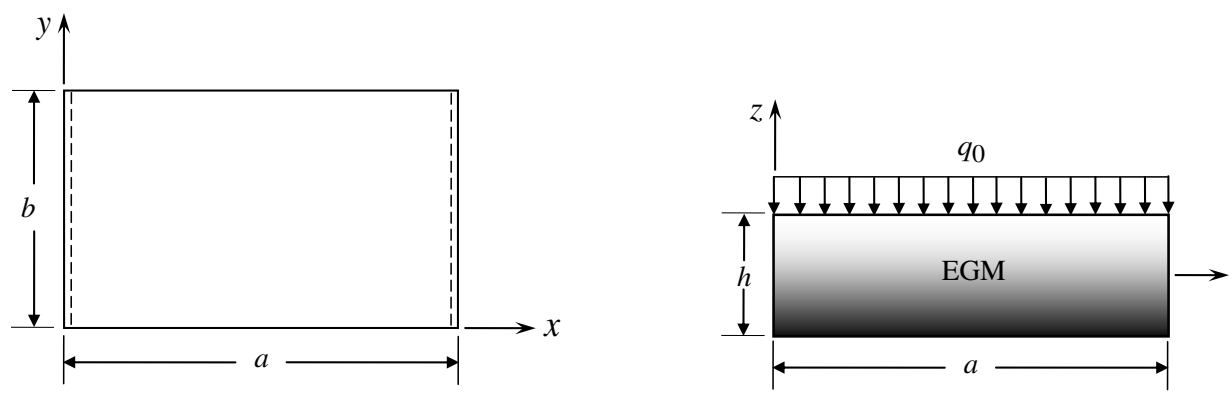

Figure 1. Geometry and coordinate system of the EGM plate under uniform load. 
where $\bar{P}_{0}$ is the corresponding property of the homogeneous plate, and $\eta$ and $k$ are geometric parameters. The value of $\eta$ equals to zero represents a fully homogeneous plate. The above exponential law assumption reflects a simple rule of mixtures applies only to the thickness direction. The power law exponents, $\eta$ and $k$, may be varied to obtain different distributions of the components materials through the thickness of the plate.

The total potential energy of the present EGM rectangular plate may be expressed as

$$
\Pi=U_{\mathrm{str}}-W_{\mathrm{ext}}-W_{\mathrm{int}},
$$

where $U_{\text {str }}$ is the strain energy of the EGM plate, $W_{\text {ext }}$ is the work of external forces, and $W_{\text {int }}$ is the work of internal forces. They are given by

$$
\begin{aligned}
U_{\mathrm{str}} & =\int_{V_{0}} \sigma_{i j} \epsilon_{i j} d V, \\
W_{\mathrm{ext}} & =\int_{S_{0}} p_{i} u_{i} d S, \\
W_{\mathrm{int}} & =\int_{V_{0}} X_{i} u_{i} d V,
\end{aligned}
$$

where $\sigma_{i j}$ is the Cauchy stress tensor, $\epsilon_{i j}$ is the small strain tensor, $p_{i}$ are the external applied loads, $u_{i}$ are the displacements in the spatial frame, and $X_{i}$ are the body forces per unit initial volume. The general governing equations of equilibrium are obtained by employing the principle of virtual displacements. The stresses and applied loads are assumed to be constants.

The displacements of a material point located at $(x, y, z)$ in the EGM plate may be written as follows [Zenkour 2004a; 2004b; 2006; 2009]:

$$
\left.\begin{array}{l}
u_{1}(x, y, z)=u-z \partial w / \partial x+\Psi(z) \phi_{1}, \\
u_{2}(x, y, z)=v-z \partial w / \partial y+\Psi(z) \phi_{2}, \\
u_{3}(x, y, z)=w
\end{array}\right\}
$$

where $u, v$, and $w$ are the displacements of the middle surface along the axes $x, y$ and $z$, respectively, and $\phi_{1}$ and $\phi_{2}$ are the rotations about the $y$ and $x$ axes and account for the effect of transverse shear. The coefficient of $\phi_{1}$ and $\phi_{2}$ which is given by $\Psi(z)$ should be odd function of $z$. All of the generalized displacements $\left(u, v, w, \phi_{1}, \phi_{2}\right)$ are functions of the $(x, y)$. The displacements of the classical thin plate theory (CPT) is obtained easily by setting $\Psi(z)=0$. The displacements of the first-order shear deformation plate theory (FPT) is obtained by setting $\Psi(z)=z$. In addition, the higher-order shear deformation plate theory (HPT) [Reddy 2000] is obtained by setting

$$
\Psi(z)=z\left[1-\frac{4}{3}\left(\frac{z}{h}\right)^{2}\right] .
$$

Also, the sinusoidal shear deformation plate theory (SPT) is obtained by setting (see [Zenkour 2004a; 2004b; 2006; 2009])

$$
\Psi(z)=\frac{h}{\pi} \sin \frac{\pi z}{h} .
$$


Note that the present SPT, as well as HPT, is simplified by enforcing traction-free boundary conditions at the plate faces. The SPT accounts according to a cosine-law distribution of the transverse shear deformation through the thickness of the EGM plate. The SPT, HPT and FPT contain the same number of dependent unknowns. No transversal shear correction factors are needed for both SPT and HPT because a correct representation of the transversal shearing strain is given.

The six strain components $\epsilon_{i j}$ compatible with the displacement field in (4) are

$$
\left\{\begin{array}{l}
\epsilon_{11} \\
\epsilon_{22} \\
\epsilon_{12}
\end{array}\right\}=\left\{\begin{array}{l}
\epsilon_{11}^{0} \\
\epsilon_{22}^{0} \\
\epsilon_{12}^{0}
\end{array}\right\}+z\left\{\begin{array}{l}
\kappa_{11} \\
\kappa_{22} \\
\kappa_{12}
\end{array}\right\}+\Psi(z)\left\{\begin{array}{l}
\theta_{11} \\
\theta_{22} \\
\theta_{12}
\end{array}\right\}, \quad \epsilon_{33}=0, \quad\left\{\begin{array}{l}
\epsilon_{23} \\
\epsilon_{13}
\end{array}\right\}=\Psi(z), 3\left\{\begin{array}{c}
\epsilon_{23}^{0} \\
\epsilon_{13}^{0}
\end{array}\right\},
$$

where

$$
\begin{gathered}
\epsilon_{11}^{0}=u, 1, \quad \epsilon_{22}^{0}=v_{, 2}, \quad \epsilon_{23}^{0}=\phi_{2}, \quad \epsilon_{13}^{0}=\phi_{1}, \quad \epsilon_{12}^{0}=v_{, 1}+u_{, 2}, \\
\kappa_{11}=-w_{, 11}, \quad \kappa_{22}=-w_{, 22}, \quad \kappa_{12}=-2 w_{, 12}, \quad \theta_{11}=\phi_{1,1}, \quad \theta_{22}=\phi_{2,2}, \quad \theta_{12}=\phi_{2,1}+\phi_{1,2} .
\end{gathered}
$$

The stress-strain relations for a linear isotropic elastic plate are given by

$$
\left\{\begin{array}{l}
\sigma_{11} \\
\sigma_{22}
\end{array}\right\}=\frac{E(z)}{1-v^{2}}\left[\begin{array}{ll}
1 & v \\
v & 1
\end{array}\right]\left\{\begin{array}{l}
\epsilon_{11}-\alpha \Delta T-\beta \Delta C \\
\epsilon_{22}-\alpha \Delta T-\beta \Delta C
\end{array}\right\}, \quad\left\{\sigma_{23}, \sigma_{13}, \sigma_{12}\right\}=\frac{E(z)}{2(1+v)}\left\{\epsilon_{23}, \epsilon_{13}, \epsilon_{12}\right\},
$$

where $E$ is Young's modulus, $v$ is Poisson's ratio, $\alpha$ and $\beta$ are the thermal and moisture expansion coefficients, $\Delta T=T-T_{0}$ in which $T$ is the applied temperature and $T_{0}$ is the reference temperature, and $\Delta C=C-C_{0}$ in which $C$ is the moisture concentration and $C_{0}$ is the reference moisture concentration.

The applied temperature distribution $T(x, y, z)$ and the moisture concentration $C(x, y, z)$ through the thickness are assumed, respectively, to be

$$
\begin{aligned}
& T(x, y, z)=T_{1}(x, y)+\frac{z}{h} T_{2}(x, y)+\frac{\Psi(z)}{h} T_{3}(x, y), \\
& C(x, y, z)=C_{1}(x, y)+\frac{z}{h} C_{2}(x, y)+\frac{\Psi(z)}{h} C_{3}(x, y) .
\end{aligned}
$$

\section{Governing equations}

The governing equations of equilibrium can be derived by using the principle of virtual displacements. The equilibrium equations associated with the present sinusoidal shear deformation theory are

$$
\begin{aligned}
N_{1,1}+N_{12,2} & =0, \\
N_{12,1}+N_{2,2} & =0, \\
M_{1,11}+2 M_{12,12}+M_{2,22}+q & =0, \\
S_{1,1}+S_{12,2}-Q_{13} & =0, \\
S_{12,1}+S_{2,2}-Q_{23} & =0,
\end{aligned}
$$

where the stress and moment resultants $\left(N_{1}, N_{2}, N_{12}, M_{1}, M_{2}, M_{12}, S_{1}, S_{2}, S_{12}, Q_{13}, Q_{23}\right)$ of the FGM 
plate can be obtained by integrating (10) over the thickness, and are written as

$$
\begin{gathered}
\left\{\begin{array}{c}
N_{i} \\
M_{i} \\
S_{i}
\end{array}\right\}=\frac{1}{1-v^{2}}\left[\begin{array}{lll}
A_{11}^{1} & A_{12}^{1} & A_{13}^{1} \\
A_{12}^{1} & A_{22}^{1} & A_{23}^{1} \\
A_{13}^{1} & A_{23}^{1} & A_{33}^{1}
\end{array}\right]\left\{\begin{array}{c}
\bar{\epsilon}_{i}^{0} \\
\bar{\kappa}_{i} \\
\bar{\theta}_{i}
\end{array}\right\}+\frac{1}{v-1}\left[\begin{array}{ccc}
A_{11}^{\alpha} & A_{12}^{\alpha} & A_{13}^{\alpha} \\
A_{12}^{\alpha} & A_{22}^{\alpha} & A_{23}^{\alpha} \\
A_{13}^{\alpha} & A_{23}^{\alpha} & A_{33}^{\alpha}
\end{array}\right]\left\{\begin{array}{l}
T_{1}^{*} \\
T_{2}^{*} \\
T_{3}^{*}
\end{array}\right\}+\frac{1}{v-1}\left[\begin{array}{lll}
A_{11}^{\beta} & A_{12}^{\beta} & A_{13}^{\beta} \\
A_{12}^{\beta} & A_{22}^{\beta} & A_{23}^{\beta} \\
A_{13}^{\beta} & A_{23}^{\beta} & A_{33}^{\beta}
\end{array}\right]\left\{\begin{array}{l}
C_{1}^{*} \\
C_{2}^{*} \\
C_{3}^{*}
\end{array}\right\}, \\
\left\{\begin{array}{c}
N_{12} \\
M_{12} \\
S_{12}
\end{array}\right\}=\frac{1}{2(1+v)}\left[\begin{array}{lll}
A_{11}^{1} & A_{12}^{1} & A_{13}^{1} \\
A_{12}^{1} & A_{22}^{1} & A_{23}^{1} \\
A_{13}^{1} & A_{23}^{1} & A_{33}^{1}
\end{array}\right]\left\{\begin{array}{l}
\epsilon_{12}^{0} \\
\kappa_{12} \\
\eta_{12}
\end{array}\right\},
\end{gathered}
$$

and

$$
\left\{\begin{array}{l}
Q_{13} \\
Q_{23}
\end{array}\right\}=\frac{B}{2(1+v)}\left\{\begin{array}{c}
\epsilon_{13}^{0} \\
\epsilon_{23}^{0}
\end{array}\right\}
$$

where $i=1,2$ and

$$
\begin{array}{rlrl}
\bar{\epsilon}_{1}^{0} & =\epsilon_{11}^{0}+v \epsilon_{22}^{0}, & \bar{\epsilon}_{2}^{0}=\epsilon_{22}^{0}+v \epsilon_{11}^{0}, \\
\bar{\kappa}_{1}=\kappa_{11}+v \kappa_{22}, & \bar{\kappa}_{2}=\kappa_{22}+v \kappa_{11}, \\
\bar{\theta}_{1}=\theta_{11}+v \theta_{22}, & \bar{\theta}_{2}=\theta_{22}+v \theta_{11}, \\
T_{1}^{*}=T_{1}-T_{0}, & C_{1}^{*}=C_{1}-C_{0}, \\
T_{j}^{*}=T_{j} / h, & C_{j}^{*}=C_{j} / h, \quad j=2,3 .
\end{array}
$$

In (13a)-(13c), $N_{1}, N_{2}$, and $N_{12}$ and $M_{1}, M_{2}$, and $M_{12}$ are the basic components of stress resultants and stress couples; $S_{1}, S_{2}$, and $S_{12}$ are additional stress couples associated with the transversal shear effects; and $Q_{13}$ and $Q_{23}$ are transversal shear stress resultants. The coefficients $A_{r s}^{\gamma}(\gamma=1, \alpha, \beta ; r, s=1,2,3)$ are defined by

$$
\begin{gathered}
\left\{A_{11}^{\gamma}, A_{12}^{\gamma}, A_{22}^{\gamma}\right\}=\int_{-h / 2}^{+h / 2} \gamma E\left\{1, z, z^{2}\right\} d z, \quad\left\{A_{13}^{\gamma}, A_{23}^{\gamma}, A_{33}^{\gamma}\right\}=\int_{-h / 2}^{+h / 2} \gamma \Psi E\{1, z, \Psi\} d z, \\
B=\bar{K} \int_{-h / 2}^{+h / 2} E\left(\Psi_{, 3}\right)^{2} d z,
\end{gathered}
$$

where $\bar{K}$ is the shear correction factor for FPT, taken to be $\frac{5}{6}$.

\section{Exact solutions for EGM plates}

The determination of transverse deflections and stresses are of fundamental importance in the design of many structural components. An exact closed-form solution to (12a)-(12e) can be constructed when the plate is of a rectangular geometry (Figure 1) with the following edge conditions, loading and displacements.

4.1. Boundary conditions. The following set of simply supported boundary conditions along the edges of the plate is considered:

$$
\begin{array}{ll}
v=w=\phi_{2}=N_{1}=M_{1}=S_{1}=0 & \text { at } x=0, a, \\
u=w=\phi_{1}=N_{2}=M_{2}=S_{2}=0 & \text { at } y=0, b,
\end{array}
$$


4.2. Loading and displacements. Rectangular plates are generally classified in accordance with the type support used in the absence of the body forces and lateral loads except the external force $q(x, y)$. We are here concerned with the exact solutions of (12a)-(12e) for simply supported FGM plate. To solve this problem, Navier presented the uniform external force and the transverse uniform temperature and moisture concentration loads in the form of a double trigonometric series

$$
\left\{\begin{array}{c}
q \\
T_{i} \\
C_{i}
\end{array}\right\}=\sum_{m, n=1,3,5, \ldots}^{\infty}\left\{\begin{array}{c}
q_{0} \\
t_{i} \\
c_{i}
\end{array}\right\} \frac{16}{m n \pi^{2}} \sin (\lambda x) \sin (\mu y), \quad(i=1,2,3),
$$

where $\lambda=m \pi / a, \mu=n \pi / b, m$ and $n$ are mode numbers, $q_{0}$ represents the intensity of the load at the plate center, $t_{i}$ and $c_{i}$ are constants.

Following the Navier solution procedure, we assume the following solution form for $\left(u, v, w, \phi_{1}, \phi_{2}\right)$ that satisfies the simply supported boundary conditions,

$$
\left\{\begin{array}{c}
u \\
v \\
w \\
\phi_{1} \\
\phi_{2}
\end{array}\right\}=\sum_{m, n=1,3,5, \ldots}^{\infty}\left\{\begin{array}{l}
U_{m n} \cos (\lambda x) \sin (\mu y) \\
V_{m n} \sin (\lambda x) \cos (\mu y) \\
W_{m n} \sin (\lambda x) \sin (\mu y) \\
X_{m n} \cos (\lambda x) \sin (\mu y) \\
Y_{m n} \sin (\lambda x) \cos (\mu y)
\end{array}\right\},
$$

where $U_{m n}, V_{m n}, W_{m n}, X_{m n}$, and $Y_{m n}$ are arbitrary parameters to be determined subjected to the condition that the solution in (18) satisfies the differential equations, (12a)-(12e). Substituting (18) into (12a)(12e), one obtains

$$
[P]\{\underline{\Delta}\}=\{F\},
$$

where $\{\underline{\Delta}\}$ and $\{F\}$ denote the columns given by

$$
\{\underline{\Delta}\}^{T}=\left\{U_{m n}, V_{m n}, W_{m n}, X_{m n}, Y_{m n}\right\}, \quad\{F\}^{T}=\left\{F_{1}, F_{2}, F_{3}, F_{4}, F_{5}\right\} .
$$

The elements $P_{i j}$ of the coefficient matrix $[P]$ and the elements $F_{i}$ of the load vector $\{F\}$ are given in the Appendix.

Moreover, substituting (7) into (10) with the help of (18), one can obtain the stress components $\sigma_{i j}$ in terms of Young's modulus and the arbitrary parameters $U_{m n}, V_{m n}, W_{m n}, X_{m n}$, and $Y_{m n}$ as follows:

$$
\begin{aligned}
\sigma_{11}=-E(z) & \sum_{m, n=1,3,5, \ldots}^{\infty}\left\{\frac{1}{1-v^{2}}\left(\lambda U_{m n}+v \mu V_{m n}-z\left(\lambda^{2}+v \mu^{2}\right) W_{m n}+\Psi(z)\left(\lambda X_{m n}+v \mu Y_{m n}\right)\right)\right. \\
& \left.+\frac{\alpha(z)}{(1-v) h}\left(h\left(t_{1}-T_{0}\right)+z t_{2}+\Psi(z) t_{3}\right)+\frac{\beta(z)}{(1-v) h}\left(h\left(c_{1}-C_{0}\right)+z c_{2}+\Psi(z) c_{3}\right)\right\} \sin (\lambda x) \sin (\mu y), \\
\sigma_{22}=-E(z) & \sum_{m, n=1,3,5, \ldots}^{\infty}\left\{\frac{1}{1-v^{2}}\left(v \lambda U_{m n}+\mu V_{m n}-z\left(v \lambda^{2}+\mu^{2}\right) W_{m n}+\Psi(z)\left(v \lambda X_{m n}+\mu Y_{m n}\right)\right)\right. \\
+ & \left.\frac{\alpha(z)}{(1-v) h}\left(h\left(t_{1}-T_{0}\right)+z t_{2}+\Psi(z) t_{3}\right)+\frac{\beta(z)}{(1-v) h}\left(h\left(c_{1}-C_{0}\right)+z c_{2}+\Psi(z) c_{3}\right)\right\} \sin (\lambda x) \sin (\mu y),
\end{aligned}
$$




$$
\begin{aligned}
\sigma_{23} & =\frac{E(z)}{2(1+v)} \sum_{m, n=1,3,5, \ldots}^{\infty} \Psi(z)_{, 3} Y_{m n} \sin (\lambda x) \cos (\mu y), \\
\sigma_{13} & =\frac{E(z)}{2(1+v)} \sum_{m, n=1,3,5, \ldots}^{\infty} \Psi(z)_{, 3} X_{m n} \cos (\lambda x) \sin (\mu y), \\
\sigma_{12} & =\frac{E(z)}{2(1+v)} \sum_{m, n=1,3,5, \ldots}^{\infty}\left(\mu U_{m n}+\lambda V_{m n}-2 z \lambda \mu W_{m n}+\Psi(z)\left(\mu X_{m n}+\lambda Y_{m n}\right)\right) \cos (\lambda x) \cos (\mu y) .
\end{aligned}
$$

\section{Numerical results}

The flexural response of EGM plates subjected to uniform transverse pressure in hygrothermal environment is studied and the results are depicted in graphical form in Figures 2-5. The material properties of the composite material are considered to be dependent on temperature and moisture. The material properties are taken in the analysis at the reference temperature $T_{0}=21^{\circ} \mathrm{C}$ (room temperature) and

\begin{tabular}{|cccccccc}
\hline$\eta$ & Theory & \multicolumn{3}{c}{$k=1$} & & & $k=2$ \\
& & $w^{*}$ & $\sigma_{1}$ & $\sigma_{5}$ & $w^{*}$ & $\sigma_{1}$ & $\sigma_{5}$ \\
0.0 & CPT & 1.37568 & 1.13905 & - & 1.37568 & 1.13905 & - \\
& FPT & 1.41072 & 1.13905 & 0.83759 & 1.41072 & 1.13905 & 0.83759 \\
& HPT & 1.41071 & 1.16220 & 1.03274 & 1.41071 & 1.16220 & 1.03274 \\
& SPT & 1.41065 & 1.16329 & 1.06316 & 1.41065 & 1.16329 & 1.06316 \\
0.5 & CPT & 1.39072 & 1.10461 & - & 1.42831 & 1.09636 & - \\
& FPT & 1.42540 & 1.10461 & 0.82893 & 1.46481 & 1.09636 & 0.87263 \\
& HPT & 1.42556 & 1.12654 & 1.06057 & 1.46418 & 1.11202 & 1.01562 \\
& SPT & 1.42552 & 1.12785 & 1.09444 & 1.46410 & 1.11235 & 1.04159 \\
1.0 & CPT & 1.43690 & 1.06625 & - & 1.48857 & 1.04696 & - \\
& FPT & 1.47052 & 1.06625 & 0.80368 & 1.52655 & 1.04696 & 0.90790 \\
& HPT & 1.47118 & 1.09251 & 1.14760 & 1.52531 & 1.05738 & 1.00654 \\
& SPT & 1.47116 & 1.09458 & 1.19207 & 1.52519 & 1.05720 & 1.02878 \\
1.5 & CPT & 1.51752 & 1.03519 & - & 1.55630 & 0.99370 & - \\
& FPT & 1.54948 & 1.03519 & 0.76393 & 1.59576 & 0.99370 & 0.94335 \\
& HPT & 1.55089 & 1.06756 & 1.30451 & 1.59391 & 1.00052 & 1.00418 \\
& SPT & 1.55092 & 1.07050 & 1.36763 & 1.59378 & 0.99999 & 1.02332 \\
2.0 & CPT & 1.63862 & 1.01769 & - & 1.63137 & 0.93864 & - \\
& FPT & 1.66843 & 1.01769 & 0.71272 & 1.67232 & 0.93864 & 0.97893 \\
& HPT & 1.67080 & 1.05580 & 1.54971 & 1.66988 & 0.94305 & 1.00744 \\
& SPT & 1.67089 & 1.05943 & 1.64112 & 1.66972 & 0.94231 & 1.02400 \\
\hline
\end{tabular}

Table 1. Effects of the exponents $k$ and $\eta$ on the deflection $w^{*}$ and stresses $\sigma_{1}$ and $\sigma_{5}$ for EGM rectangular plate using various theories $\left(t_{2}=10, c_{2}=1, t_{3}=c_{3}=0\right)$. 
moisture concentration $C_{0}=0 \%$ as follows:

$$
E=3.45 \mathrm{GPa}, \quad v=0.35, \quad \alpha=72.0 \times 10^{-6} /{ }^{\circ} \mathrm{C}, \quad \beta=0.33 .
$$

Many examples have been solved numerically using the following fixed data: (unless otherwise stated) $q_{0}=100, a=10 h, b=2 a, k=2, t_{1}=t_{2}=0, c_{1}=c_{2}=0$. The plate is assumed to be simply supported on all four edges. Numerical results are presented in terms of nondimensional stresses and deflection. The various nondimensional parameters used are

$$
\begin{gathered}
w^{*}=\frac{10^{2} D}{a^{4} q_{0}} w\left(\frac{a}{2}, \frac{b}{2}\right), \quad \sigma_{1}=\frac{1}{10^{2} q_{0}} \sigma_{11}\left(\frac{a}{2}, \frac{b}{2}, \frac{z}{h}\right), \quad \sigma_{5}=-\frac{1}{10 q_{0}} \sigma_{13}\left(0, \frac{b}{2}, \frac{z}{h}\right), \\
\sigma_{6}=\frac{1}{10^{2} q_{0}} \sigma_{12}\left(0,0, \frac{z}{h}\right), \quad D=\frac{h^{3} E_{0}}{12\left(1-v^{2}\right)} .
\end{gathered}
$$

The longitudinal stress $\sigma_{1}$, the transverse shear stress $\sigma_{5}$ and the in-plane shear stress $\sigma_{6}$ are computed at $z=h / 2, z=0$ and $z=-h / 2$, respectively. For the sake of completeness, Table 1 on the previous page shows results of the present sinusoidal plate theory (SPT) are compared with those obtained using HPT,
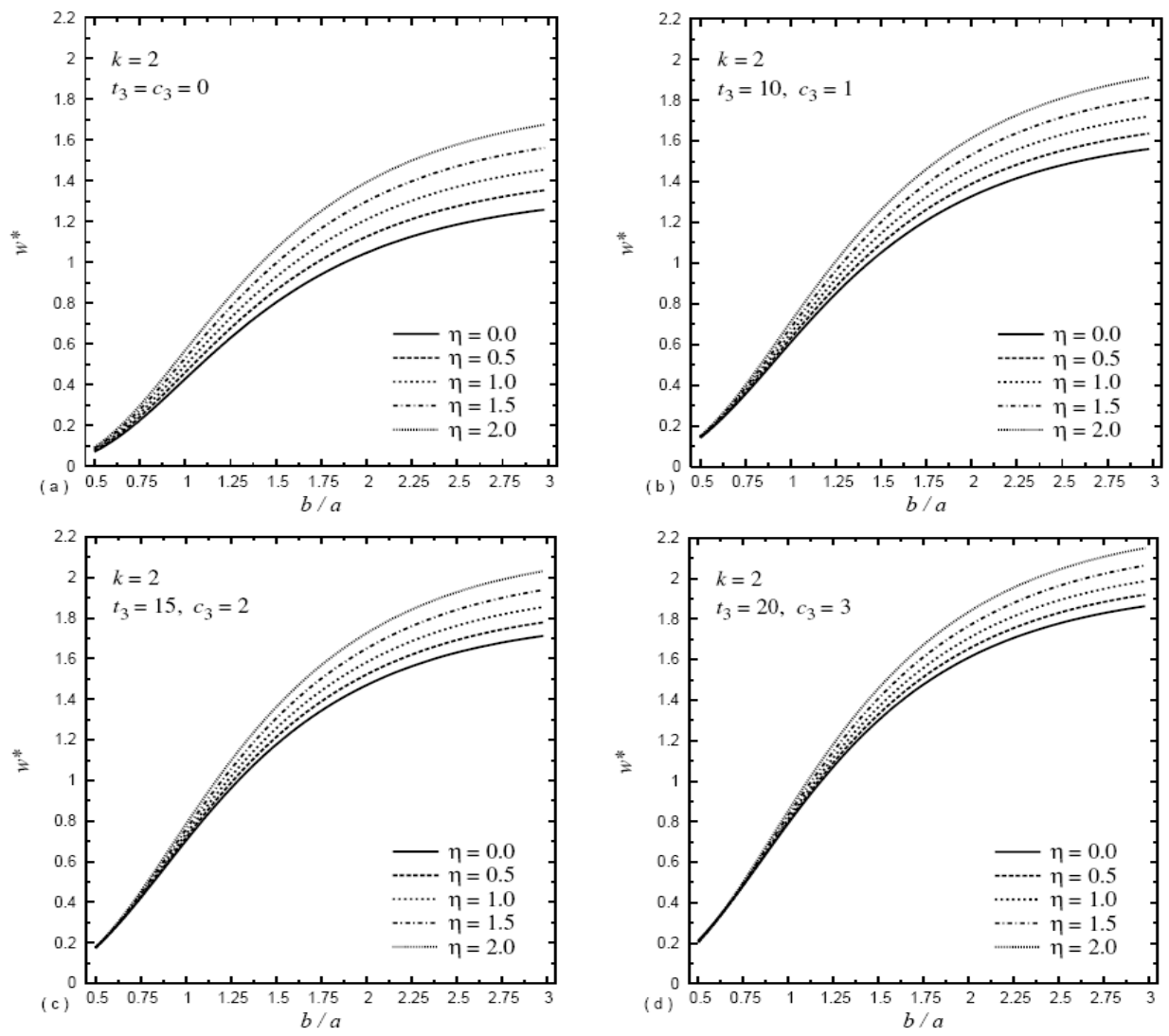

Figure 2. Dimensionless center deflection $w^{*}$ versus the aspect ratio $b / a$ of an EGM plate for various values of the power-law exponent $\eta$ : (a) $t_{3}=c_{3}=0$; (b) $t_{3}=10, c_{3}=1$; (c) $t_{3}=15, c_{3}=2$; (d) $t_{3}=20, c_{3}=3$. 
FPT, and CPT for the deflection $w^{*}$, the in-plane longitudinal stress $\sigma_{1}$ and the transverse shear stress $\sigma_{5}$ in bending of EGM plates under uniformly distributed loading. The deflection $w^{*}$ increases with an increase in the exponent $k$ or in $\eta$. The stresses $\sigma_{1}$ and $\sigma_{5}$ increase as $k$ increases, but decrease as $\eta$ increases. It is to be noted that the CPT gives the same in-plane longitudinal stress $\sigma_{1}$ as that of the FPT.

The effects of temperature, moisture concentration and their combination on the nondimensional bending response of the EGM plate are shown in Figures 2-5. The effect of the EG parameter $\eta$ on the center deflection and stresses for different values of the thermal and moisture concentration parameters is investigated. The variation of the center deflection versus the aspect ratio $b / a$ for homogeneous $(\eta=0)$ and EGM plates is presented in Figure 2. It is observed that central deflection increases with increase in moisture concentration, temperature and increase in both simultaneously. The increase is highest when hygrothermal condition is taken and it is least when only effect of temperature is considered. It is also noted that the deflection increases with the increase of $b / a$ and $\eta$.

The distribution of the in-plane longitudinal stress $\sigma_{1}$ through-the-thickness of the homogeneous $(\eta=0)$ and EGM plates is displayed in Figure 3. The longitudinal stress is no longer linear throughthe-thickness of the EGM plates in the absence of the hygrothermal parameters in which the stresses
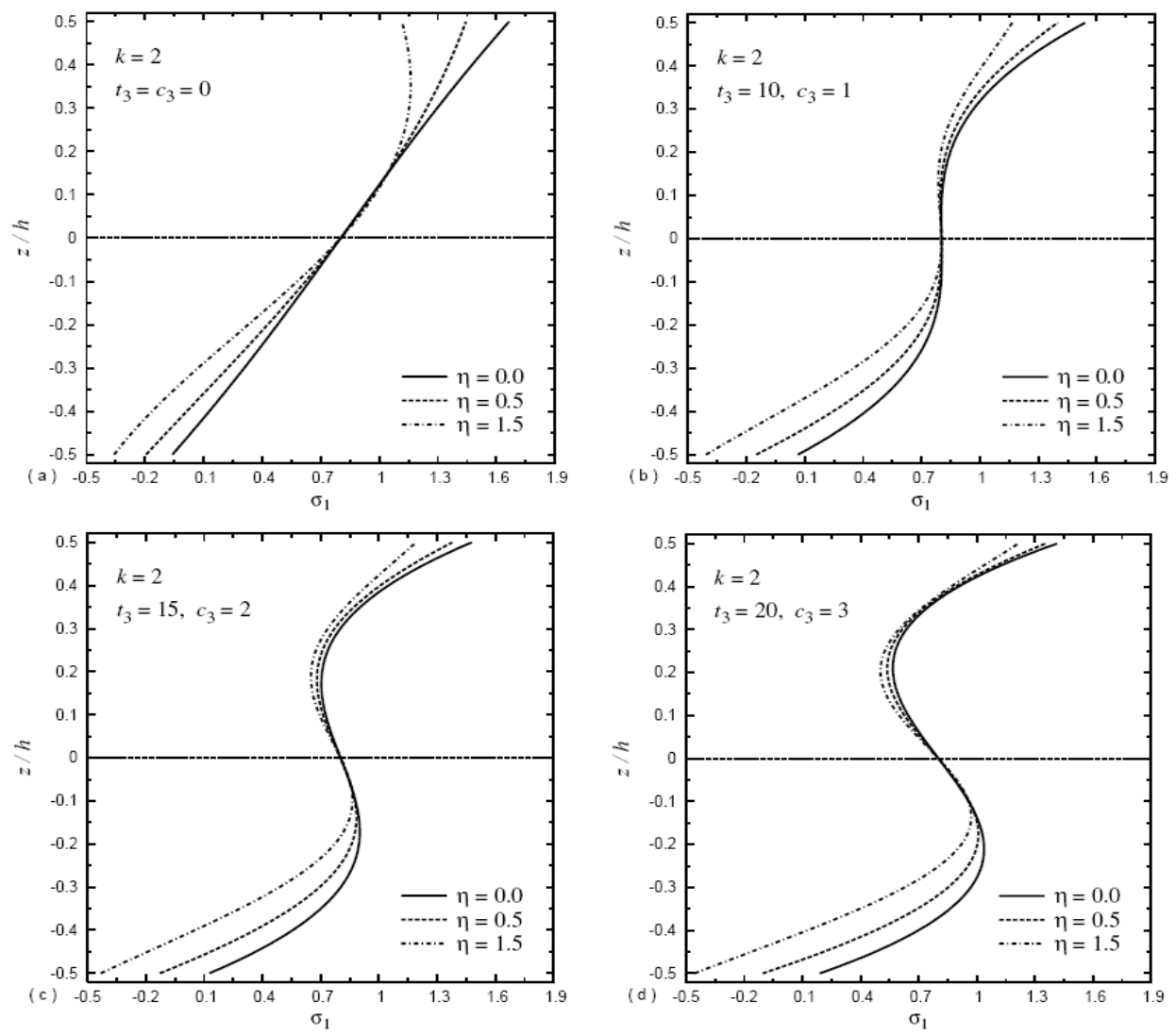

Figure 3. Dimensionless in-plane normal stress $\sigma_{1}$ through-the-thickness of a rectangular plate $(b / a=2)$ for various values of the power-law exponent $\eta$ : (a) $t_{3}=c_{3}=0$; (b) $t_{3}=10, c_{3}=1$; (c) $t_{3}=15, c_{3}=2$; (d) $t_{3}=20, c_{3}=3$. 
still tensile through the plate thickness. The longitudinal stress becomes compressive near the bottom base of the plate, especially for $\eta \neq 0$. The behavior of stress is very sensitive to the variation of $\eta$. The homogeneous plate yields the maximum longitudinal stress through-the-thickness of the plate and this irrespective of the values of the hygrothermal parameters.

The distribution of the transverse shear stress $\sigma_{5}$ through-the-thickness of the homogeneous $(\eta=0)$ and EGM plates is displayed in Figure 4. The minimum shear stress occurs at the mid-plane of the homogeneous and EGM plates and the stress still symmetric through the plate thickness. In the absence of the hygrothermal parameters the shear stress is maximum for homogeneous plate in the interval $-0.21 \leq$ $z / h \leq 0.21$. This interval is decreased to be $-0.13 \leq z / h \leq 0.13$ when $t_{3}=10$ and $c_{3}=1$. For other cases $\left(t_{3}=15, c_{3}=2\right.$ and $\left.t_{3}=20, c_{3}=3\right)$, the shear stress of the homogeneous plate is the smallest one.

Figure 5 show that the in-plane shear stress $\sigma_{6}$ is linearly distributed through-the-thickness of the homogeneous plate irrespective of the thermal or moisture concentration parameters. Otherwise, $\sigma_{6}$ is very sensitive to the variation of $\eta, t_{3}$ and $c_{3}$. This stress is tensile near the bottom surface of the plate while it is compressive near the top surface of the plate. It is symmetric through-the-thickness of the
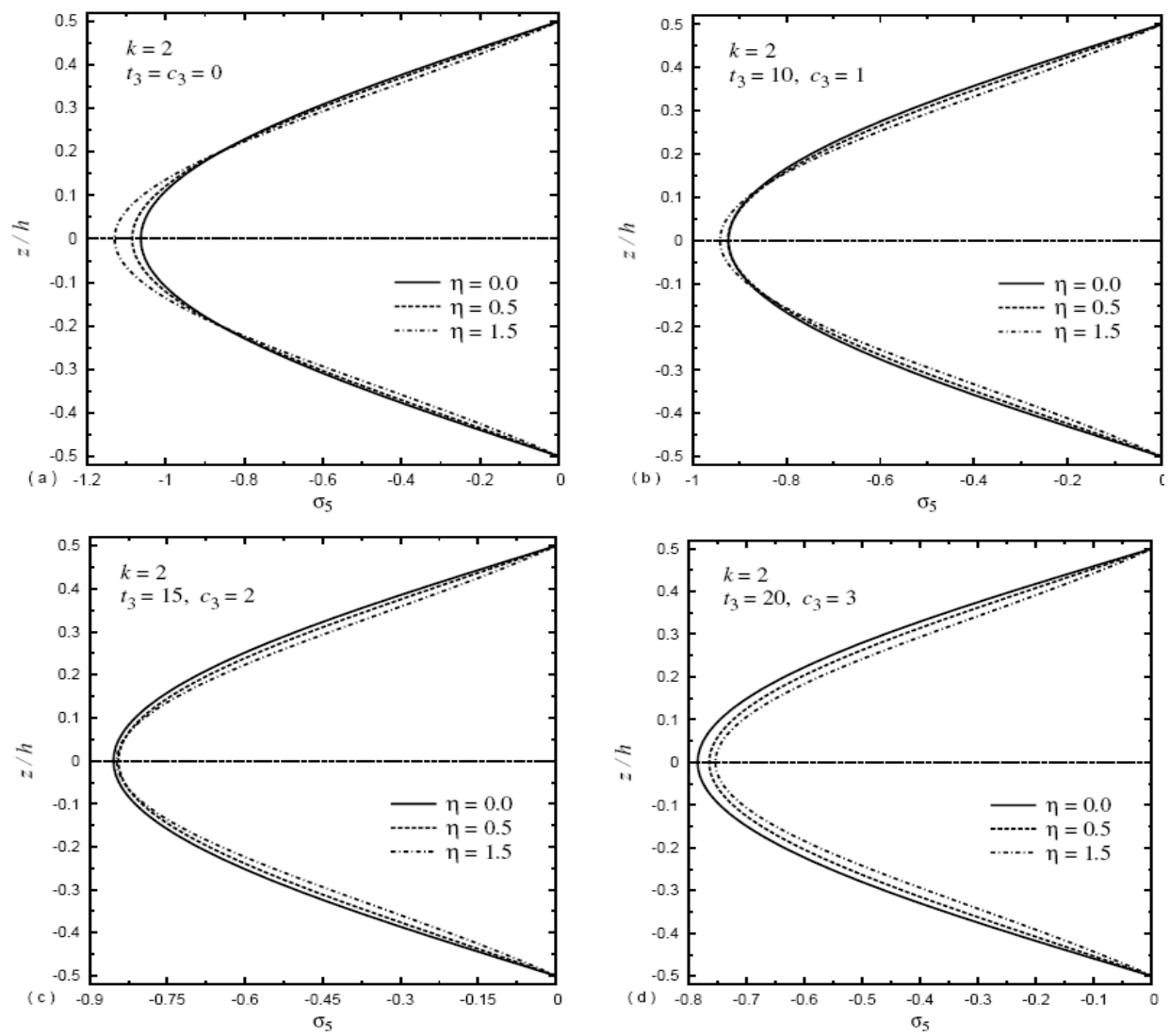

Figure 4. Dimensionless transverse shear stress $\sigma_{5}$ through-the-thickness of a rectangular plate $(b / a=2)$ for various values of the power-law exponent $\eta$ : (a) $t_{3}=c_{3}=0$; (b) $t_{3}=10, c_{3}=1 ;$ (c) $t_{3}=15, c_{3}=2 ;$ (d) $t_{3}=20, c_{3}=3$. 

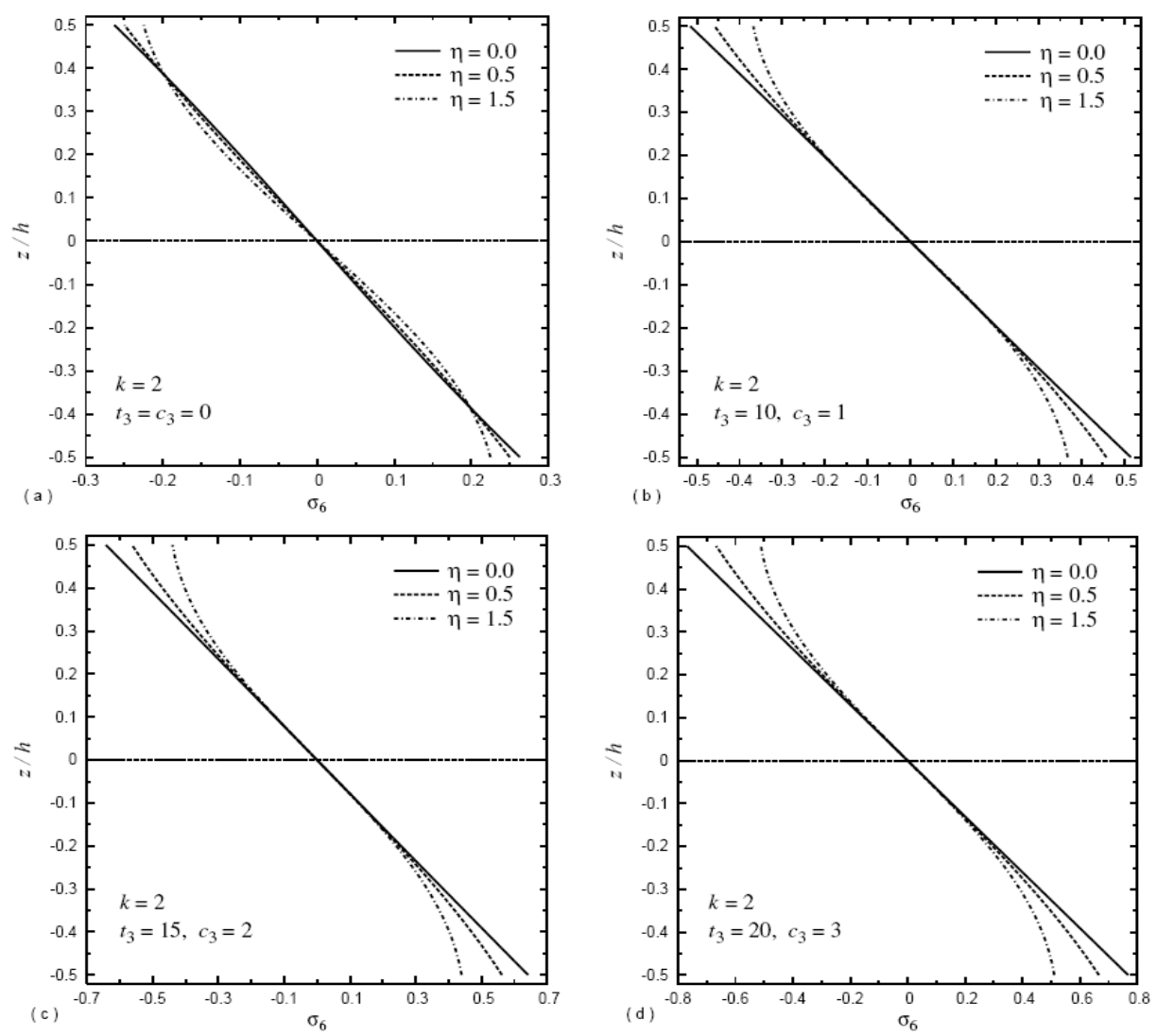

Figure 5. Dimensionless in-plane shear stress $\sigma_{6}$ through-the-thickness of a rectangular plate $(b / a=2)$ for various values of the power-law exponent $\eta$ : (a) $t_{3}=c_{3}=0$; $t_{3}=10, c_{3}=1 ;$ (c) $t_{3}=15, c_{3}=2 ;$ (d) $t_{3}=20, c_{3}=3$.

plate and vanished at its mid-plane. It is observed that this stress increases with increase in moisture concentration, temperature and increase in both simultaneously.

\section{Conclusion}

The hygrothermal effects of homogeneous and EGM plates under uniformly distributed load are presented based on the sinusoidal shear deformation theory. The present analysis includes the effects of temperature and moisture concentration on the material properties. Numerical results show that due to the change in material properties caused by a rise of temperature and a change of moisture concentration, the relationship between the hygrothermal response of homogeneous and EGM plates and the rise of temperature and/or moisture concentrations is no longer linear. The results presented herein show that the deflection and stresses will degrade with increasing moisture concentrations and temperatures. They also confirm that the characteristics of stresses and deflection are significantly influenced by temperature rise, the degree of moisture concentration, plate aspect ratio, as well as the EGM coefficient. 


\section{Appendix}

The elements $P_{i j}=P_{j i}$ of the coefficient matrix $[P]$ in (19) are given by

$$
\begin{array}{ll}
P_{11}=A_{11}^{1}\left[2 \lambda^{2}+(1-v) \mu^{2}\right], & P_{12}=\lambda \mu A_{11}^{1}(1+v), \\
P_{13}=-2 \lambda A_{12}^{1}\left(\lambda^{2}+\mu^{2}\right), & P_{14}=A_{13}^{1}\left[2 \lambda^{2}+(1-v) \mu^{2}\right], \\
P_{15}=P_{24}=\lambda \mu A_{13}^{1}(1+v), & P_{22}=A_{11}^{1}\left[(1-v) \lambda^{2}+2 \mu^{2}\right], \\
P_{23}=-2 \mu A_{12}^{1}\left(\lambda^{2}+\mu^{2}\right), & P_{25}=A_{13}^{1}\left[(1-v) \lambda^{2}+2 \mu^{2}\right], \\
P_{33}=2 A_{22}^{1}\left(\lambda^{2}+\mu^{2}\right)^{2}, & P_{34}=-2 \lambda A_{23}^{1}\left(\lambda^{2}+\mu^{2}\right), \\
P_{35}=-2 \mu A_{23}^{1}\left(\lambda^{2}+\mu^{2}\right), & P_{44}=A_{33}^{1}\left[2 \lambda^{2}+(1-v) \mu^{2}\right]+B(1-v), \\
P_{45}=\lambda \mu A_{33}^{1}(1+v), & P_{55}=A_{33}^{1}\left[(1-v) \lambda^{2}+2 \mu^{2}\right]+B(1-v) .
\end{array}
$$

The elements $F_{i}$ of the load vector $\{F\}$ in (19) are given by

$$
\begin{aligned}
& F_{1}=-2 \lambda(1+v)\left(A_{11}^{\alpha} t_{1}+A_{11}^{\beta} c_{1}+\frac{1}{h}\left(A_{12}^{\alpha} t_{2}+A_{13}^{\alpha} t_{3}+A_{12}^{\beta} c_{2}+A_{13}^{\beta} c_{3}\right)\right), \\
& F_{2}=-2 \mu(1+v)\left(A_{11}^{\alpha} t_{1}+A_{11}^{\beta} c_{1}+\frac{1}{h}\left(A_{12}^{\alpha} t_{2}+A_{13}^{\alpha} t_{3}+A_{12}^{\beta} c_{2}+A_{13}^{\beta} c_{3}\right)\right), \\
& F_{3}=2(1+v)\left\{\left(A_{12}^{\alpha} t_{1}+A_{12}^{\beta} t_{1}+\frac{1}{h}\left(A_{22}^{\alpha} t_{2}+A_{23}^{\alpha} t_{3}+A_{22}^{\beta} c_{2}+A_{23}^{\beta} c_{3}\right)\right)\left(\lambda^{2}+\mu^{2}\right)+q_{m n}(1-v)\right\}, \\
& F_{4}=-2 \lambda(1+v)\left(A_{13}^{\alpha} t_{1}+A_{13}^{\beta} c_{1}+\frac{1}{h}\left(A_{23}^{\alpha} t_{2}+A_{33}^{\alpha} t_{3}+A_{23}^{\beta} c_{2}+A_{33}^{\beta} t_{3}\right)\right), \\
& F_{5}=-2 \mu(1+v)\left(A_{13}^{\alpha} t_{1}+A_{13}^{\beta} c_{1}+\frac{1}{h}\left(A_{23}^{\alpha} t_{2}+A_{33}^{\alpha} t_{3}+A_{23}^{\beta} c_{2}+A_{33}^{\beta} c_{3}\right)\right) .
\end{aligned}
$$

\section{Acknowledgement}

This paper was funded by the Deanship of Scientific Research (DSR), King Abdulaziz University, Jeddah, under grant no. 360/130/1431. The author, therefore, acknowledges with thanks DSR technical and financial support.

\section{References}

[Adams and Miller 1977] D. F. Adams and A. K. Miller, "Hygrothermal micro stress in a unidirectional composite exhibiting inelastic materials behavior", J. Compos. Mater. 11 (1977), 285-299.

[Benkhedda et al. 2008] A. Benkhedda, A. Tounsi, and E. A. Adda Bedia, "Effect of temperature and humidity on transient hygrothermal stresses during moisture desorption in laminated composite plates", Compos. Struct. 82:4 (2008), 629-635.

[Brunelle and Robertson 1974] E. J. Brunelle and S. R. Robertson, "Initially stressed Mindlin plates", AIAA J. 12 (1974), 1036-1045.

[Brunelle and Robertson 1976] E. J. Brunelle and S. R. Robertson, "Vibrations of an initially stressed thick plate", J. Sound Vib. 45:3 (1976), 405-416.

[Herrmann and Armenakas 1962] G. Herrmann and A. E. Armenakas, "Vibrations and stability of plates under initial stress", Trans. ASCE 127:1 (1962), 458-491.

[Ishikawa et al. 1978] T. Ishikawa, K. Koyama, and S. Kobayayaski, "Thermal expansion coefficients of unidirectional composites", J. Compos. Mater. 12 (1978), 153-168. 
[Lee et al. 1992] S. Y. Lee, C. J. Chou, J. L. Jang, and J. S. Lim, "Hygrothermal effects on the linear and nonlinear analysis of symmetric angle-ply laminated plates", Compos. Struct. 21 (1992), 41-48.

[Lo et al. 2010] S. H. Lo, W. Zhen, Y. K. Cheung, and C. Wanji, "Hygrothermal effects on multilayered composite plates using a refined higher order theory", Compos. Struct. 92 (2010), 633-646.

[Patel et al. 2002] B. P. Patel, M. Ganapathi, and D. P. Makhecha, "Hygrothermal effects on the structural behaviour of thick composite laminates using higher-order theory", Compos. Struct. 56 (2002), 25-34.

[Pipes et al. 1976] R. B. Pipes, J. R. Vinson, and T. W. Chou, "On the hygrothermal response of laminated composite systems", J. Compos. Mater. 10 (1976), 129-148.

[Rao and Sinha 2004] V. V. S. Rao and P. K. Sinha, "Bending characteristic of thick multidirectional composite plates under hygrothermal environment", J. Reinf. Plast. Compos. 23 (2004), 1481-1495.

[Reddy 1997] J. N. Reddy, Mechanics of laminated composite plates, CRC Press, Boca Raton, FL, 1997.

[Reddy 2000] J. N. Reddy, “Analysis of functionally graded plates”, Int. J. Numer. Methods Eng. 47 (2000), 663-684.

[Sai Ram and Sinha 1991] K. S. Sai Ram and P. K. Sinha, "Hygrothermal effects on the bending characteristics on laminated composite plates", Comput. Struct. 40 (1991), 1009-1015.

[Sai Ram and Sinha 1992] K. S. Sai Ram and P. K. Sinha, "Hygrothermal effects on the free vibration of laminated composite plates", J. Sound Vib. 158 (1992), 133-148.

[Shen 2001] H. S. Shen, "Hygrothermal effects on the postbuckling of shear deformable laminated plates", Int. J. Mech. Sci. 43 (2001), 1259-1281.

[Strife and Prewo 1979] J. R. Strife and K. M. Prewo, "The thermal expansion behavior of unidirectional and bidirectional Kevlar/epoxy composites”, J. Compos. Mater. 13 (1979), 264-267.

[Wang et al. 2005] X. Wang, K. Dong, and X. Y. Wang, "Hygrothermal effect on dynamic interlaminar stresses in laminated plates with piezoelectric actuators", Compos. Struct. 71 (2005), 220-228.

[Whitney and Ashton 1971] J. M. Whitney and J. E. Ashton, "Effect of environment on the elastic response of layered composite plates", AIAA J. 9 (1971), 1708-1713.

[Yang and Shieh 1987] I. H. Yang and J. A. Shieh, "Vibrations of initially stressed thick, rectangular, orthotropic plates", J. Sound Vib. 119 (1987), 545-558.

[Zenkour 2004a] A. M. Zenkour, "Buckling of fiber-reinforced viscoelastic composite plates using various plate theories", $J$. Eng. Math. 50 (2004), 75-93.

[Zenkour 2004b] A. M. Zenkour, "Thermal effects on the bending response of fiber-reinforced viscoelastic composite plates using a sinusoidal shear deformation theory", Acta Mech. 171 (2004), 171-187.

[Zenkour 2005] A. M. Zenkour, "Analytical solutions for rotating exponentially-graded annular disks with various boundary conditions”, Int. J. Struct. Stab. Dyn. 5:4 (2005), 557-577.

[Zenkour 2006] A. M. Zenkour, "Generalized shear deformation theory for bending analysis of functionally graded plates", Appl. Math. Model. 30 (2006), 67-84.

[Zenkour 2009] A. M. Zenkour, "The refined sinusoidal theory for FGM plates on elastic foundations", Int. J. Mech. Sci. 51 (2009), 869-880.

[Zenkour 2010] A. M. Zenkour, "Hygro-thermo-mechanical effects on FGM plates resting on elastic foundations", Compos. Struct. 93 (2010), 234-238.

Received 16 Mar 2012. Revised 9 Jul 2012. Accepted 10 Jul 2012.

ASHRAF M. ZENKOUR: zenkour@gmail.com

Mathematics Department, King Abdulaziz University, Faculty of Science, P.O. Box 80203, Jeddah 21589, Saudi Arabia and

Department of Mathematics, Faculty of Science, Kafrelsheikh University, Kafr El-Sheikh 33516, Egypt 


\title{
JOURNAL OF MECHANICS OF MATERIALS AND STRUCTURES
}

\author{
jomms.net
}

\author{
Founded by Charles R. Steele and Marie-Louise Steele \\ EDITORS \\ Charles R. STEele \\ DAVIDE BIGONI \\ Stanford University, USA \\ University of Trento, Italy \\ IWONA JASIUK University of Illinois at Urbana-Champaign, USA \\ YASUHIDE SHINDO Tohoku University, Japan
}

\section{EDITORIAL BOARD}

H. D. Bui École Polytechnique, France

J. P. CARTER University of Sydney, Australia

R. M. CHRISTENSEN Stanford University, USA

G. M. L. GLadWELL University of Waterloo, Canada

D. H. Hodges Georgia Institute of Technology, USA

J. Hutchinson Harvard University, USA

C. HwU National Cheng Kung University, Taiwan

B. L. KARIHALOO University of Wales, UK

Y. Y. KIM Seoul National University, Republic of Korea

Z. Mroz Academy of Science, Poland

D. PAmplona Universidade Católica do Rio de Janeiro, Brazil

M. B. RUBIN Technion, Haifa, Israel

A. N. SHUPIKOV Ukrainian Academy of Sciences, Ukraine

T. TARNAI University Budapest, Hungary

F. Y. M. WAN University of California, Irvine, USA

P. WRIGGERS Universität Hannover, Germany

W. YANG Tsinghua University, China

F. ZIEGLER Technische Universität Wien, Austria

PRODUCTION production@msp.org

SILVIO LEVY Scientific Editor

See http://jomms.net for submission guidelines.

JoMMS (ISSN 1559-3959) is published in 10 issues a year. The subscription price for 2012 is US $\$ 555 /$ year for the electronic version, and \$735/year (+\$60 shipping outside the US) for print and electronic. Subscriptions, requests for back issues, and changes of address should be sent to Mathematical Sciences Publishers, Department of Mathematics, University of California, Berkeley, CA 94720-3840.

JoMMS peer-review and production is managed by EditFLow ${ }^{\circledR}$ from Mathematical Sciences Publishers.

\author{
mathematical sciences publishers \\ http://msp.org/ \\ A NON-PROFIT CORPORATION \\ Typeset in $\mathrm{IAT}_{\mathrm{E}} \mathrm{X}$ \\ Copyright $@ 2012$ by Mathematical Sciences Publishers
}




\section{Journal of Mechanics of Materials and Structures}

\section{Volume 7, No. $7 \quad$ September 2012}

Molecular dynamics-based continuum models for the linear elasticity of nanofilms and nanowires with anisotropic surface effects

Wonbae Kim, Seung Yun Rhee and Máenghyo Cho

Characterization of human skin through skin expansion

Djenane C. Pamplona and Claudio R. Carvalho 641

In-plane dynamic excitation of AAC masonry walls patched with FRP: dynamic testing and analysis

DVIR ELMALICH and ODED RABINOVITCH

Hygrothermal analysis of exponentially graded rectangular plates

ASHRAF M. ZENKOUR 687

Green's function for symmetric loading of an elastic sphere with application to contact problems ALEXeY S. TITOVICH and ANDREW N. NORRIS 701

Multi-hit armour characterisation of metal-composite bi-layers

K. Karthikeyan, B. P. Russell, V. S. Deshpande and N. A. Fleck 